\title{
Aberrant anti-viral response of natural killer cells in severe asthma
}

\author{
Justine Devulder (10 ${ }^{1}$, Cécile Chenivesse ${ }^{1,2}$, Valérie Ledroit ${ }^{1}$, Stéphanie Fry ${ }^{1,2}$, \\ Pierre-Emmanuel Lobert ${ }^{3}$, Didier Hober ${ }^{3}$, Anne Tsicopoulos ${ }^{1,2}$ and \\ Catherine Duez (10 1
}

Affiliations: 'Univ. Lille, CNRS, Inserm, CHU Lille, Institut Pasteur de Lille, U1019 - UMR 8204 - CIIL Center for Infection and Immunity of Lille, Lille, France. ${ }^{2} \mathrm{CHU}$ Lille, Service de Pneumologie et ImmunoAllergologie, Centre de compétence pour les Maladies Pulmonaires Rares, Lille, France. ${ }^{3}$ Univ. Lille, CHU Lille, EA 3610 - Pathogenèse virale du diabète de type 1, Lille, France.

Correspondence: Catherine Duez, Center for Infection and Immunity of Lille, Pulmonary Immunity Team, Institut Pasteur de Lille, 1 rue du Pr Calmette, BP 245, 59019, Lille Cedex, France. E-mail: catherine.duezla inserm.fr

@ERSpublications

NK cells from severe asthma patients exhibit decreased activation, cytotoxic capacity and IFN- $\gamma$ production after in vitro stimulation with rhinovirus $\mathrm{RV}-\mathrm{A} 9$, in comparison to healthy donors. Exhausted phenotype is associated to increased Tim-3 expression. http://bit.ly/2UFjtdc

Cite this article as: Devulder J, Chenivesse C, Ledroit V, et al. Aberrant anti-viral response of natural killer cells in severe asthma. Eur Respir J 2020; 55: 1802422 [https://doi.org/10.1183/13993003.02422-2018].

ABSTRACT Rhinovirus infections are the main cause of asthma exacerbations. As natural killer (NK) cells are important actors of the antiviral innate response, we aimed at evaluating the functions of NK cells from severe asthma patients in response to rhinovirus-like molecules or rhinoviruses.

Peripheral blood mononuclear cells from patients with severe asthma and healthy donors were stimulated with pathogen-like molecules or with the rhinoviruses (RV)-A9 and RV-2. NK cell activation, degranulation and interferon (IFN) $-\gamma$ expression were analysed.

NK cells from severe asthma patients were less cytotoxic than those from healthy donors in response to toll-like receptor (TLR)3, TLR7/8 or RV-A9 but not in response to RV-2 stimulation. Furthermore, when cultured with interleukin (IL)-12+IL-15, cytokines which are produced during viral infections, NK cells from patients with severe asthma were less cytotoxic and expressed less IFN- $\gamma$ than NK cells from healthy donors. NK cells from severe asthmatics exhibited an exhausted phenotype, with an increased expression of the checkpoint molecule Tim-3.

Together, our findings indicate that the activation of NK cells from patients with severe asthma may be insufficient during some but not all respiratory infections. The exhausted phenotype may participate in NK cell impairment and aggravation of viral-induced asthma exacerbation in these patients. 


\section{Introduction}

Asthma is a chronic inflammatory disease characterised by airway inflammation and hyperresponsiveness [1]. Worldwide, 300 million people have asthma, with 5-10\% suffering from severe asthma. According to the European Respiratory Society and the American Thoracic Society, severe asthma requires treatment with high doses of inhaled corticosteroids and a second controller for more than $50 \%$ of the previous year to prevent it from becoming uncontrolled or which remains uncontrolled despite this therapy [2]. Severe asthma is associated with poor quality of life, frequent hospitalisations and mortality due to severe asthma exacerbations [3-5]. Asthma exacerbation is defined as the deterioration in the patient's symptoms requiring the use of systemic corticosteroids and/or hospitalisation to prevent a serious outcome [6]. Approximately $50-80 \%$ of asthma exacerbations are associated with respiratory viral infections (in adults and children respectively). Among viruses implicated, $50-60 \%$ are rhinoviruses (RV) $[7,8]$. Mechanisms underlying virus-induced asthma exacerbations remain poorly understood, even though defective innate immunity in response to respiratory viruses has been highlighted [9]. For example, production of interleukin (IL)-15 by alveolar macrophages after in vitro RV infection is impaired in asthma patients, and inversely correlates with the severity of lower respiratory symptoms following RV infection in vivo [10]. $\mathrm{RV}$ activates the innate immune system through several pattern recognition receptors (PRR). The capsid is recognised by toll-like receptor (TLR)2, single stranded RNA and double stranded RNA from rhinoviruses are recognised by TLR3, TLR7/8, retinoic acid-inducible gene (RIG)-1 and melanoma differentiation-associated protein (MDA)-5. Engagement of these receptors induces cytokine expression including type I and type III interferons (IFNs), but also IL-6, IL-12, and IL-15 [11].

Natural killer (NK) cells are important actors of the antiviral innate immune response [12]. Their activation depends on the integration of multiple signals from innate receptors. Some receptors recognise major histocompatibility complex class (MHC)-I and inhibit NK cell activation, whereas others recognise cytokines (like IL-12, IL-15 and IL-18), chemokines, and microbial components leading to the activation of NK cells $[13,14]$. NK cells also express several PRR including TLR2, TLR3, TLR4, TLR7/8, TLR9, nucleotide-binding oligomerization domain 2 (NOD2), NOD-, LRR- and pyrin domain-containing protein 3 (NLRP3) and RIG-I. TLR are known to activate NK cell functions either directly or in cooperation with accessory cells in a cytokine or cell-to-cell contact-dependent manner [15]. Human NK cells are classically identified by their surface expression of cluster of differentiation (CD)56 and lack of CD3, and can be categorised into two subsets, CD56 ${ }^{\text {bright }}$ and CD56 ${ }^{\text {dim }}$ NK cells [16].

The involvement of NK cells in the asthmatic disease remains unclear. Modifications in human NK cell phenotype and functional capacities have been observed in asthma, like increased CD69 and NKG2D expression by peripheral blood NK cells from patients with severe asthma $[17,18]$. In asthmatic children, decreased expression of intercellular adhesion molecule 1 (ICAM-1) and CD62L has been observed on peripheral blood NK cells during acute exacerbation [19]. NK cells may regulate eosinophilic inflammation by inducing eosinophil activation and apoptosis [20], but eosinophil apoptosis is reduced in patients with severe asthma [17]. Mouse models of allergic asthma highlight a complex role of NK cells, which may participate [21, 22] or not [23] in allergic inflammation or may resolve it [24-26]. In a mouse model of asthma exacerbation with a TLR3 agonist, NK cells expressed IL-17A and increased lung inflammation [27].

According to the hypothesis which stipulates that defective innate immunity may lead to virus-induced exacerbation of severe asthma, we aimed to analyse NK cell functions and investigated their activation, cytotoxicity and cytokine production following microbial compound stimulation in vitro. We compared the functions of peripheral blood NK cells from severe asthma patients with healthy donors. We show that NK cells from severe asthma patients are hyporesponsive to in vitro stimulation with TLR agonists and human rhinovirus RV-A9. Our exploratory study may help define a novel endotype of severe asthma, which should be confirmed by analysing a larger cohort of patients.

\section{Materials and methods \\ Patients}

Patient's clinical data are described in table 1. All patients were non-smoker or ex-smoker with a cumulative smoking exposure below 10 pack-years. More detailed information can be found as supplementary material.

\section{Peripheral blood mononuclear cells and NK cell isolation}

Peripheral blood mononuclear cells (PBMC) were isolated from heparinised venous blood and NK cells were purified by immunomagnetic separation (STEMCELL Technologies, Vancouver, Canada). Further methodological details are described in the supplementary material. 
TABLE 1 Patient characteristics

\begin{tabular}{lcc} 
& Healthy donors & Severe asthma patients \\
\hline Subjects $\mathbf{n}$ & 11 & 31 \\
Age years & $38(24-61)$ & $57(19-78)$ \\
Females $\%$ & 64 & 58 \\
BMI $>\mathbf{3 0} \mathbf{~ k g} \cdot \mathbf{m}^{-\mathbf{2}} \%$ & 0 & 47.2 \\
ACT $>\mathbf{2 0} \%$ & $\mathrm{NA}$ & 17.7 \\
Exacerbations in the last year & $\mathrm{NA}$ & $4(0-12)$ \\
FEV $\mathbf{1} \%$ predicted & $100(95-105)$ & $68(34-112)$ \\
Blood eosinophils per $\mathbf{m m}^{\mathbf{3}}$ & $0(0-168)$ & $350(0-1320)$ \\
Atopy $\%$ & 0 & 35.4 \\
Sinusitis \% & 0 & 9.5 \\
GORD \% & 0 & 26.8 \\
Long-term OCS \% & 0 & 45.2 \\
\hline
\end{tabular}

Data are presented as median (range), unless otherwise stated. ACT: asthma control test; $\mathrm{FEV}_{1}$ : forced expiratory volume in $1 \mathrm{~s}$; GORD: gastro-oesophageal reflux disease; OCS: oral corticosteroids; NA: nonapplicable. ${ }^{\#}$ : based on positive skin test.

\section{Rhinoviruses production}

Rhinovirus A9 (RV-A9, American Type Culture Collection (ATCC) VR-489) and rhinovirus 2 (RV-2, ATCC VR-482) were propagated in H1-HeLa cells. The infectious titre of virus stock was $1.44 \times 10^{7} \mathrm{TCID} 50 \cdot \mathrm{mL}^{-1}$, as detailed in the supplementary material.

\section{Cell stimulation}

PBMC or purified NK cells were stimulated with IL-12+IL-15, or with an agonist for TLR3: polyinosinic-polycytidylic acid (poly(I:C)); for TLR9: ODN 2395; for TLR7/8: Resiquimod (R848); for TLR2/6: FSL1; for NKp46: haemagglutinin A of influenza A (HA), with RV-A9 or RV-2 during $24 \mathrm{~h}$. Concentrations and manufacturers are provided in supplementary material. For the analysis of NK cell degranulation, PBMC were cultured with K562 myeloid tumour cells (ATCC) at a ratio of 100 PBMC for 1 K562 for $3 \mathrm{~h}$.

\section{Flow cytometry analysis}

After stimulation, PBMC were stained with the viability marker and antibodies for extracellular and intracellular targets. Further methodological details are described in the supplementary material.

\section{Cytokine and chemokine measurement}

CXCL10, CXCL9, granzyme B, and IFN- $\gamma$ were measured in culture supernatants by Luminex assay (Life Technologies, Carlsbad, CA, USA) or ELISA (R\&D Systems, Minneapolis, MN, USA) according to the manufacturer's instructions. More cytokines were measured as shown in the supplementary material.

\section{Statistical analysis}

Statistical analysis was performed using GraphPad Prism software. All results are expressed as median (min-max). Comparisons between stimuli (figs. 1 and 2) were performed using two-way analysis of variance (ANOVA) with Dunnett's correction for multiple comparisons. Comparisons between stimuli (figs. 3-5) and between patient's groups were assessed by two-way ANOVA followed by Bonferroni's post-tests. Wilcoxon or Mann-Whitney tests were used for comparison between staining Tim-3 and control antibody, and between patient's groups, respectively (fig. 6). The significance level was set at $\mathrm{p}<0.05$. Analysis of statistical power was performed using $\mathrm{G}^{*}$ Power software (version 3.1.9.4), and $\alpha$ error probability set to 0.05 .

\section{Results}

Severe asthma is associated with decreased NK cell activation and cytotoxicity in response to PBMC stimulation with pathogen-like molecules

The effect of PBMC stimulation on NK cell activation was assessed by the expression of CD69 (fig. 1a). Following the stimulation with the cytokine cocktail IL-12+IL-15, or with poly(I:C) (TLR3 agonist), R848 (TLR7/8 agonist) and CpG (TLR9 agonist), the proportion of $\mathrm{CD} 9^{+} \mathrm{NK}$ cells from healthy donors significantly increased compared to the medium condition, suggesting that NK cells were activated. Similarly, NK cells from patients with severe asthma were activated by IL-12+IL-15, R848 and CpG 

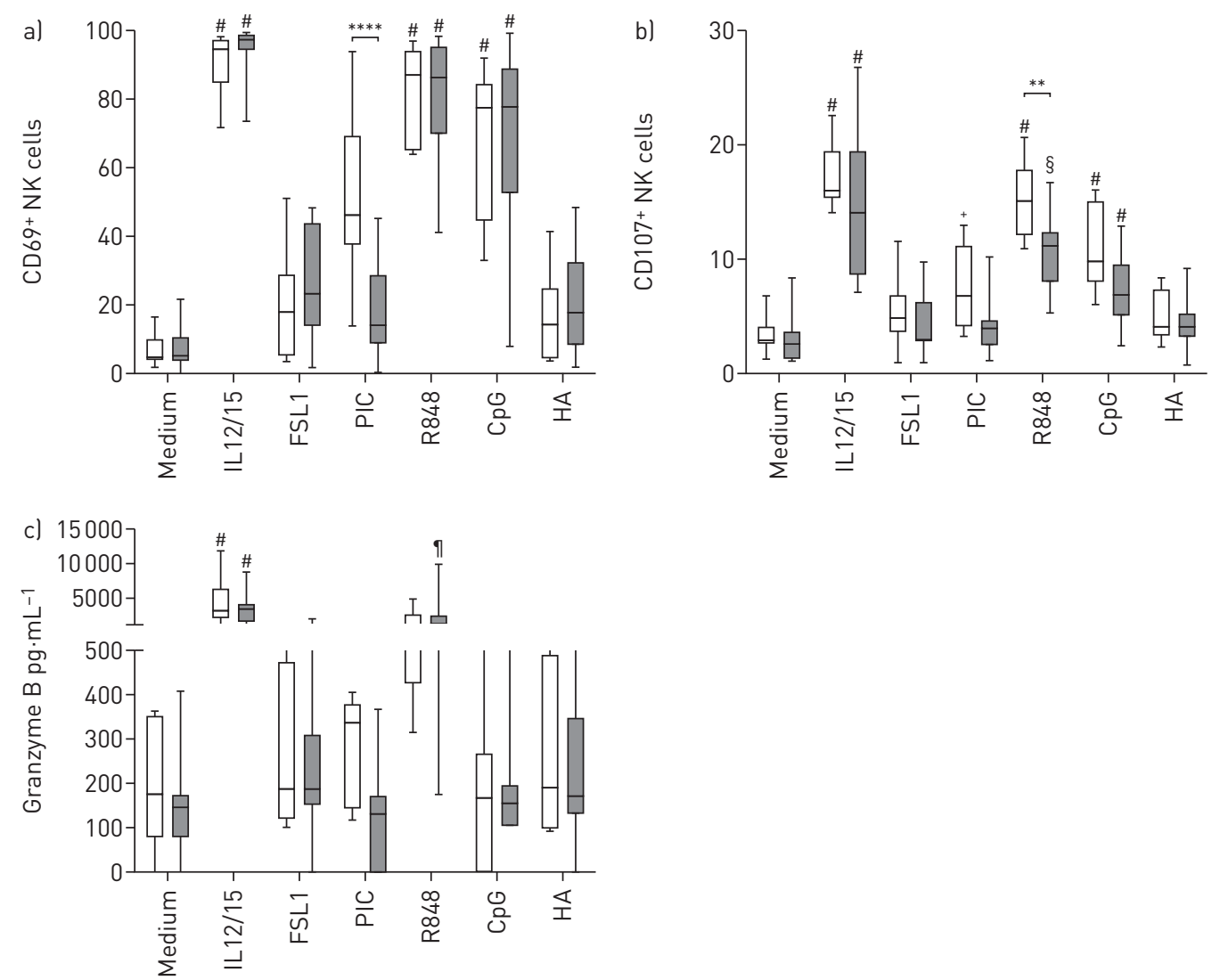

FIGURE 1 Natural killer (NK) cells from severe asthma patients exhibit a defective activation following peripheral blood mononuclear cell (PBMC) stimulation with molecules mimicking microbial compounds. PBMC from severe asthma patients and healthy donors were stimulated with interleukin (IL)-12+IL-15, FSL1 (Toll-like receptor (TLR)2/6 agonist), polyinosinic-polycytidylic acid (poly(I:C); TLR3 agonist), resiquimod (R848, TLR7/8 agonist), CpG oligonucleotides (CpG, TLR9 agonist), or haemagglutinin A of influenza A (HA; recognised by NKp46) for $24 \mathrm{~h}$. a) Percentage of CD69 and b) CD107a-positive NK cells. c) Concentration of Granzyme B in PBMC supernatants. Horizontal lines represent the median, boxes the interquartile range and whiskers the range. Statistical comparisons between medium condition and stimulated conditions were performed with two-way ANOVA followed by Dunnett's post-test $\left(^{\#}: p<0.0001,{ }^{\S}: p<0.01,{ }^{+}: p<0.05\right)$. Healthy donors ( $n=8$, white) and severe asthma patients ( $n=14$, grey) were compared with two-way ANOVA followed by Bonferroni's post-test $\left.{ }^{* *}: p<0.01,{ }^{* * * *}: p<0.0001\right)$.

stimulation. However, in response to poly(I:C), the percentage of $\mathrm{CD} 69^{+} \mathrm{NK}$ cells was significantly lower in patients with severe asthma than in healthy donors.

The cytotoxicity of NK cells was evaluated with the expression of CD107a and the release of granzyme B in PBMC supernatants after culture with K562 target cells (figure $1 \mathrm{~b}$ and c). After PBMC stimulation with IL-12+IL-15, poly(I:C), R848 and CpG, NK cells from healthy donors showed signs of degranulation as the proportion of $\mathrm{CD} 107 \mathrm{a}^{+} \mathrm{NK}$ cells significantly increased compared to the medium condition. The concentration of granzyme B in supernatants was significantly increased in response to IL-12+IL-15, and not significantly in response to poly(I:C) and R848. Similarly, NK cells from patients with severe asthma exhibited signs of degranulation in response to IL-12+IL-15, R848 and CpG. In contrast, the percentage of $\mathrm{CD} 107 \mathrm{a}^{+} \mathrm{NK}$ cells significantly decreased in response to R848 and non-significantly to poly(I:C) and CpG in patients with severe asthma compared with healthy donors. The concentration of granzyme B seemed to be diminished in the supernatants of PBMC from patients with severe asthma after poly(I:C) but no other stimulation. No differences were observed between different phenotypes of severe asthma, such as allergic versus non-allergic asthma, eosinophilic versus non-eosinophilic asthma, exacerbation prone or not. No difference was seen either when comparing severe asthma patients treated or not with oral corticotherapy (figure S2) or with body mass index (BMI) less than or greater than to $30 \mathrm{~kg} \cdot \mathrm{m}^{-2}$ (figure S3). However, due to a lack of statistical power (0.075 and 0.099 for CD69, 0.052 and 0.091 for CD107, and 0.051 and 0.171 for IFN- $\gamma$, for separation based on corticotherapy or BMI respectively), we cannot conclude to a true absence of difference. Altogether, these results indicate that NK cells from severe asthma patients have impaired activation and cytotoxicity in response to poly(I:C) and R848. 

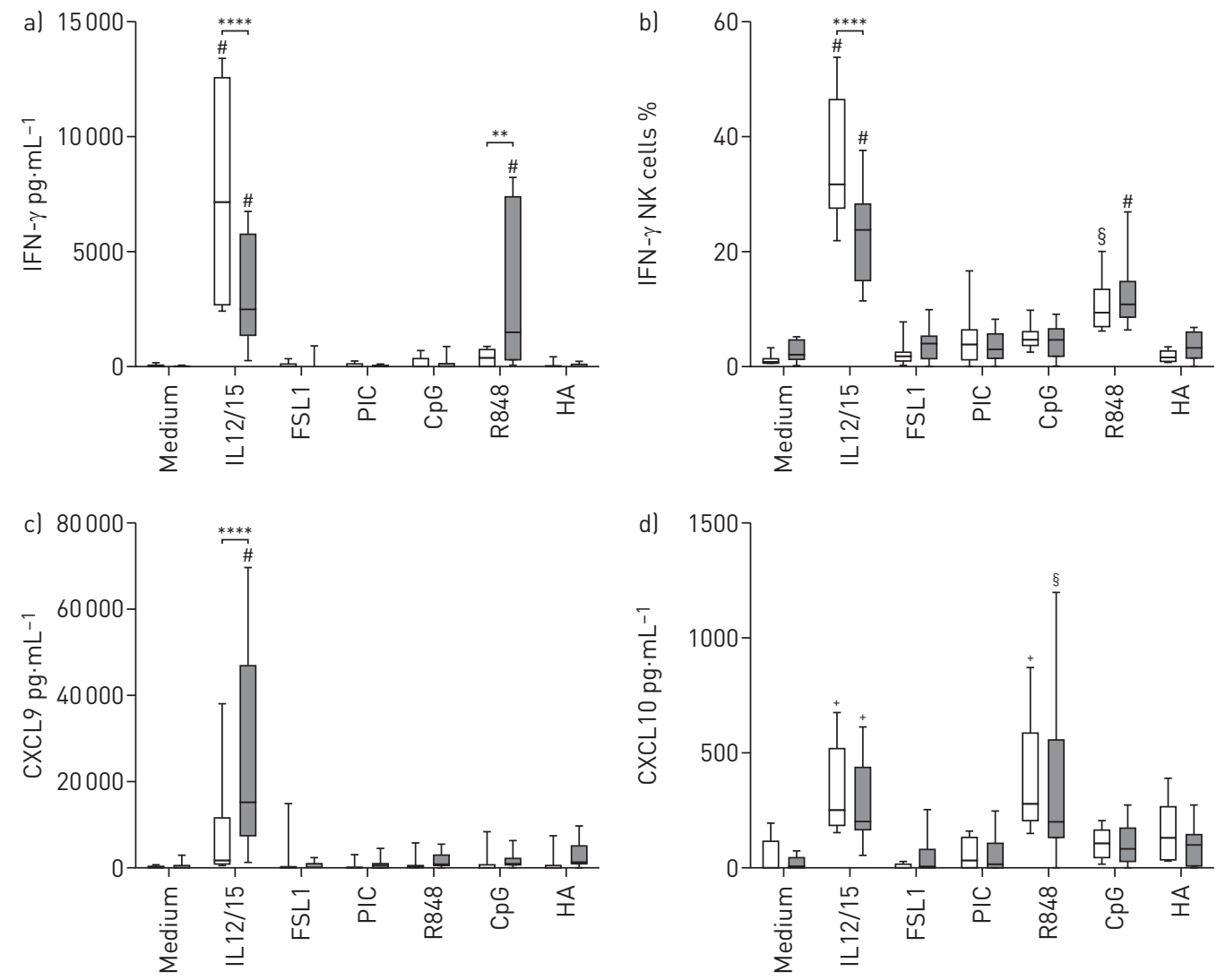

FIGURE 2 Decreased production of interferon (IFN)- $\gamma$ following peripheral blood mononuclear cell (PBMC) stimulation with interleukin (IL)-12+IL-15 but increased production of CXCL9 in severe asthma patients. PBMC from severe asthma patients and healthy donors were stimulated with interleukin (IL)-12+IL-15, FSL1 (TLR2/6 agonist), polyinosinic-polycytidylic acid (poly(I:C), TLR3 agonist), resiquimod (R848, TLR7/8 agonist), CpG oligonucleotides (CpG, TLR9 agonist), or haemagglutinin A of influenza A (HA; recognised by NKp46) for $24 \mathrm{~h}$. a) Concentrations of IFN- $\gamma\left(\mathrm{pg} \cdot \mathrm{mL}^{-1}\right)$ in supernatant. b) Intracellular expression of IFN- $\gamma$ in natural killer (NK) cells expressed as percentage of positive NK cells. c) Concentration of CXCL9 and d) CXCL10 (pg. $\left.\mathrm{mL}^{-1}\right)$. Horizontal lines represent the median, boxes represent the interquartile range and whiskers represent the range. Statistical comparisons between medium condition and stimulated conditions were performed with two-way ANOVA followed by Dunnett's post-test $\left(^{\#}: p<0.0001,{ }^{\S}: p<0.001,{ }^{+}: p<0.05\right)$. Healthy donors $(n=8$, white) and severe asthma patients ( $n=14$, grey) were compared with two-way ANOVA followed by Bonferroni's post-test (**: $\left.p<0.01,{ }^{* * * *}: p<0.0001\right)$.

Severe asthma is associated with decreased IFN- $\gamma$ production by NK cells in response to stimulation with IL-12+IL-15

IFN- $\gamma$ is the main cytokine expressed and rapidly produced by NK cells following their activation. PBMC stimulation with IL-12+IL-15 significantly increased the release of IFN- $\gamma$ (figure $2 \mathrm{a}$ ), as well as interferon-inducible chemokines, CXCL9 and CXCL10 (figure $2 \mathrm{c}$ and $\mathrm{d}$ ). However, although IFN- $\gamma$ release by PBMC from severe asthma patients was significantly lower compared with healthy donors, levels of CXCL9 and CXCL10 were increased or unchanged, respectively. While no other stimulation induced CXCL9, CXCL10 was induced by CpG stimulation, similarly in both patients, suggesting differential regulation of these two interferon-inducible chemokines. NK cells were one of the cellular sources of IFN- $\gamma$, as IL-12+IL-15 stimulation significantly increased the percentage of IFN $-\gamma^{+} \mathrm{NK}$ cells both in healthy and severe asthma patients (figure 2b), although the percentage of IFN- $\gamma^{+} \mathrm{NK}$ cells was significantly lower in severe asthma patients than in controls. However, while R848 significantly increased IFN- $\gamma$ secretion by PBMC only in patients with severe asthma, IFN- $\gamma^{+} \mathrm{NK}$ cells were increased in both type of donors, suggesting that other cells produced IFN- $\gamma$ after PBMC stimulation with R848. The increased expression and production of IFN- $\gamma$ after R848 stimulation, whereas CXCL9 and CXCL10 were not increased, strengthen the idea that the latter were independent of IFN- $\gamma$. Overall, these results show that NK cells from patients with severe asthma may also be deficient in their ability to produce the anti-viral cytokine IFN- $\gamma$. 

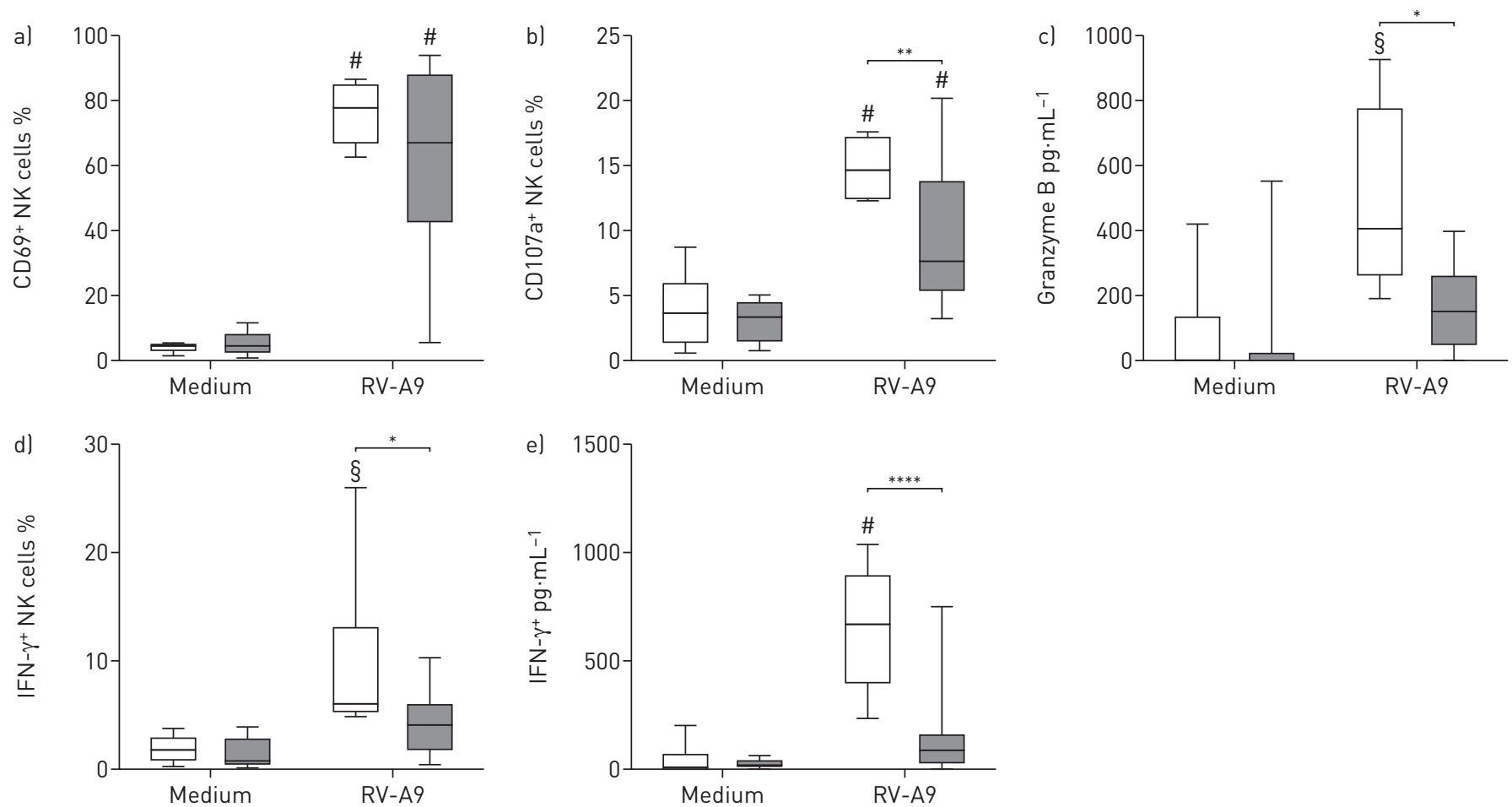

FIGURE 3 Natural killer (NK) cells from severe asthma patients exhibit decreased cytotoxic status and interferon (IFN)- $\gamma$ expression following peripheral blood mononuclear cell (PBMC) stimulation with rhinovirus RV-A9. PBMC from healthy donors ( $\mathrm{n}=6$, white) and severe asthma patients ( $n=9$, grey) were stimulated with RV-A9. a) percentage of CD69 and b) CD107a-positive NK cells. c) Granzyme B secretion in PBMC supernatants. d) Percentage of IFN- $\gamma+\mathrm{NK}$ cells and e) concentration of IFN- $\gamma$ in PBMC supernatant. Horizontal lines represent the median, boxes represent the interquartile range and whiskers represent the range. Statistical comparisons between medium and RV-A9 condition were performed with two-way ANOVA followed by Bonferroni's post-test ( $\#$ : $p<0.0001, \S: p<0.01$ ). Healthy donors and severe asthma patients were compared with two-way ANOVA followed by Bonferroni's post-test (****: $p<0.0001,{ }^{* *}: p<0.01, *: p<0.05$ ).

Severe asthma is associated with deficient NK cells response to in vitro RV-A9 stimulation To closely mimic the pathophysiological conditions, PBMC were stimulated with live rhinovirus A9 (RV-A9), which belongs to the major group of rhinovirus. RV-A9 significantly increased the proportion of $\mathrm{CD} 9^{+} \mathrm{NK}$ cells both in healthy donors and patients with severe asthma (figure 3a); however, the percentage of $\mathrm{CD} 9^{+} \mathrm{NK}$ cells in severe asthma patients was slightly lower compared with healthy donors. Purified NK cells were not activated by RV-A9, suggesting that other cells activated by RV-A9 are

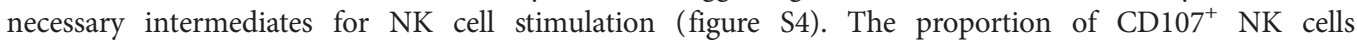
significantly increased following the stimulation of PBMC with RV-A9 in both groups, indicating that NK
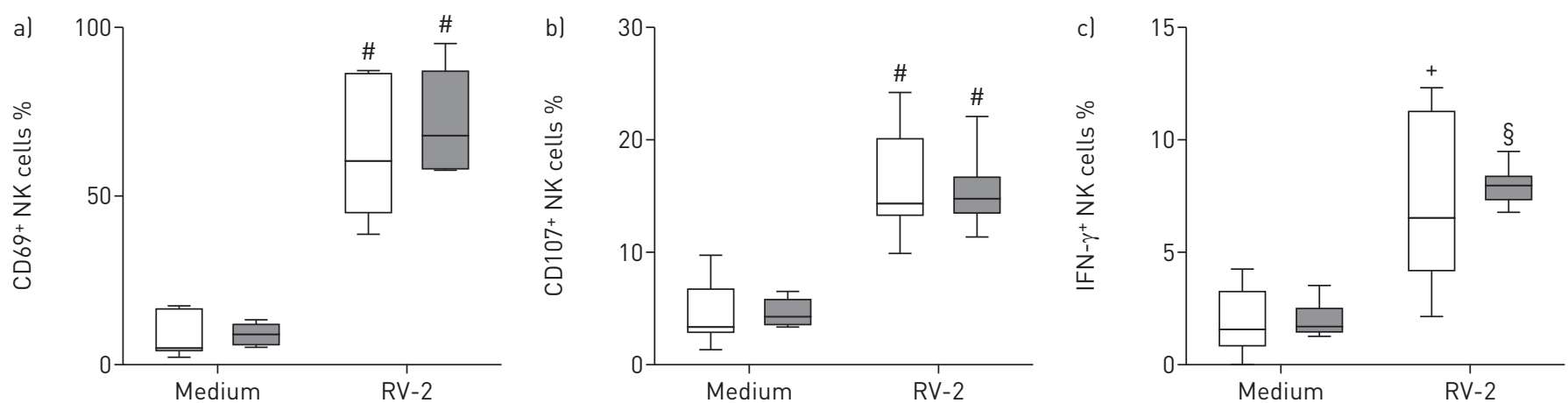

FIGURE 4 Natural killer (NK) cells from severe asthma patients does not exhibit decreased cytotoxic status and interferon (IFN)- $\gamma$ expression following peripheral blood mononuclear cell (PBMC) stimulation with RV-2. PBMC from healthy donors ( $n=7$, white) and severe asthma patients $(n=5$, grey) were stimulated with RV-2. a) percentage of CD69, b) CD107a and c) IFN- $\gamma$ positive NK cells. Horizontal lines represent the median, boxes represent the interquartile range and whiskers represent the range. Statistical comparisons between medium and RV-2 condition were performed with two-way ANOVA followed by Bonferroni's post-test $\left(^{\#}: p<0.0001,{ }^{\S}: p<0.01,{ }^{+}: p<0.05\right)$. Healthy donors and severe asthma patients were compared with two-way ANOVA followed by Bonferroni's post-test (NS: non-significant). 


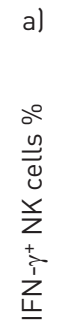

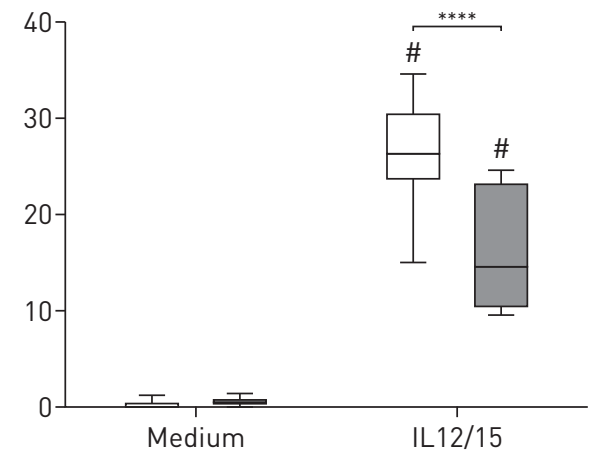

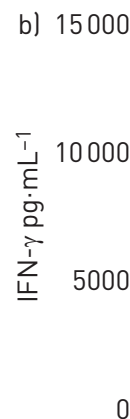

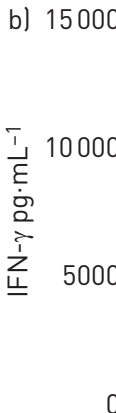

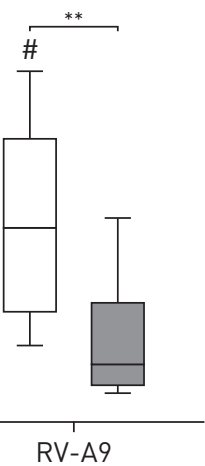

FIGURE 5 Defect of interferon (IFN)- $\gamma$ expression and secretion by natural killer (NK) cells from severe asthma patients after direct stimulation with interleukin (IL)-12+IL-15. NK cells from healthy donors ( $n=8$, white) and severe asthma patients ( $n=9$, grey) were purified and stimulated with IL-12+IL-15. a) Percentage of IFN- $\gamma+\mathrm{NK}$ cells and b) concentration of IFN- $\gamma$ in NK cell supernatant. Horizontal lines represent the median, boxes represent the interquartile range and whiskers represent the range. Statistical comparisons between medium and IL12/15 condition were performed with two-way ANOVA followed by Dunnett's post-test (\#: $p<0.0001)$. Healthy donors and severe asthma patients were compared with two-way ANOVA followed by Bonferroni's post-test $\left({ }^{* * * *}: p<0.0001,{ }^{* *}: p<0.01\right)$.

cells were sufficiently activated to degranulate after target cell encounter. In parallel, granzyme B release increased in PBMC supernatants (figure 3c). In severe asthma patients, the proportion of $\mathrm{CD} 107^{+} \mathrm{NK}$ cells and the concentration of granzyme B were significantly lower compared to healthy donors (figure $3 \mathrm{~b}$ and c). Similarly, RV-A9 increased IFN- $\gamma$ expression by NK cells and IFN- $\gamma$ production in both groups, but significantly less in severe asthma patients (figure $3 \mathrm{~d}$ and e). Altogether, these results suggest that NK cells from severe asthma patients are deficient in their activation, cytotoxicity, and IFN- $\gamma$ production in response to RV-A9. However, when PBMC from severe asthma patients were activated with another rhinovirus (RV-2), which belong to the minor group of rhinovirus, NK cells exhibited activation, cytotoxicity and IFN- $\gamma$ production similarly to NK cells from healthy donors (figure 4). This latter result suggests that RV-A9 and RV-2 induced differential response in NK cells from severe asthma patients and that hyporesponsiveness to viruses is a specific but not universal feature of NK cells in severe asthma.

\section{NK cells from severe asthma patients exhibit an exhausted phenotype}

Because RV-A9 stimulation of NK cells is indirect, we wondered if NK cells from severe asthma patients were deficient or responded to a deficient activation. IL-12+IL-15 can directly stimulate purified NK cells, and indeed increased the proportion of IFN- $\gamma^{+}$NK cells (figure 5a) and IFN- $\gamma$ secretion by purified NK cells from both donor types (figure 5b). However, both IFN- $\gamma^{+} \mathrm{NK}$ cells and IFN- $\gamma$ concentration were significantly decreased in severe asthma patients compared to healthy subjects, suggesting that NK cells from severe asthma patients present an intrinsic defect leading to a decreased expression and production of IFN- $\gamma$. Therefore, although several other cells may behave abnormally in severe asthma patients in response to RV-A9, NK cells per se exhibit aberrant functions.

An increasing number of studies suggest that NK cells can become functionally exhausted during chronic infections. The checkpoint molecules or exhaustion markers NKG2A and programmed cell death protein 1 (PD-1) were detected on NK cells but their expression was not modified between healthy donors and severe asthma patients (figure S5). In contrast, Tim3 expression by NK cells was significantly increased in severe asthma patients compared with healthy donors (figure 6a-e). This was restricted to NK cells as Tim3 expression by $\mathrm{CD}^{+} \mathrm{T}$ cells was identical in healthy donors and severe asthma patients (figure 6f-h). Tim3 expression on NK cells did not increase after in vitro treatment of NK cells with dexamethasone (figure S6), suggesting that the observed increased expression of Tim3 on NK cells from severe asthma patients may be independent of inhaled corticosteroid treatment. Therefore, increased Tim 3 expression suggests that NK cells from severe asthma patients may be exhausted, which may partially account for their impaired functions in response to TLR agonists and RV-A9 stimulation.

\section{Discussion}

In this study, we explored NK cell functions in severe asthma patients and healthy donors in response to stimulation either with molecules mimicking viruses or with a live virus. We chose to stimulate mononuclear cells in order to allow accessory cells to interact with NK cells. 

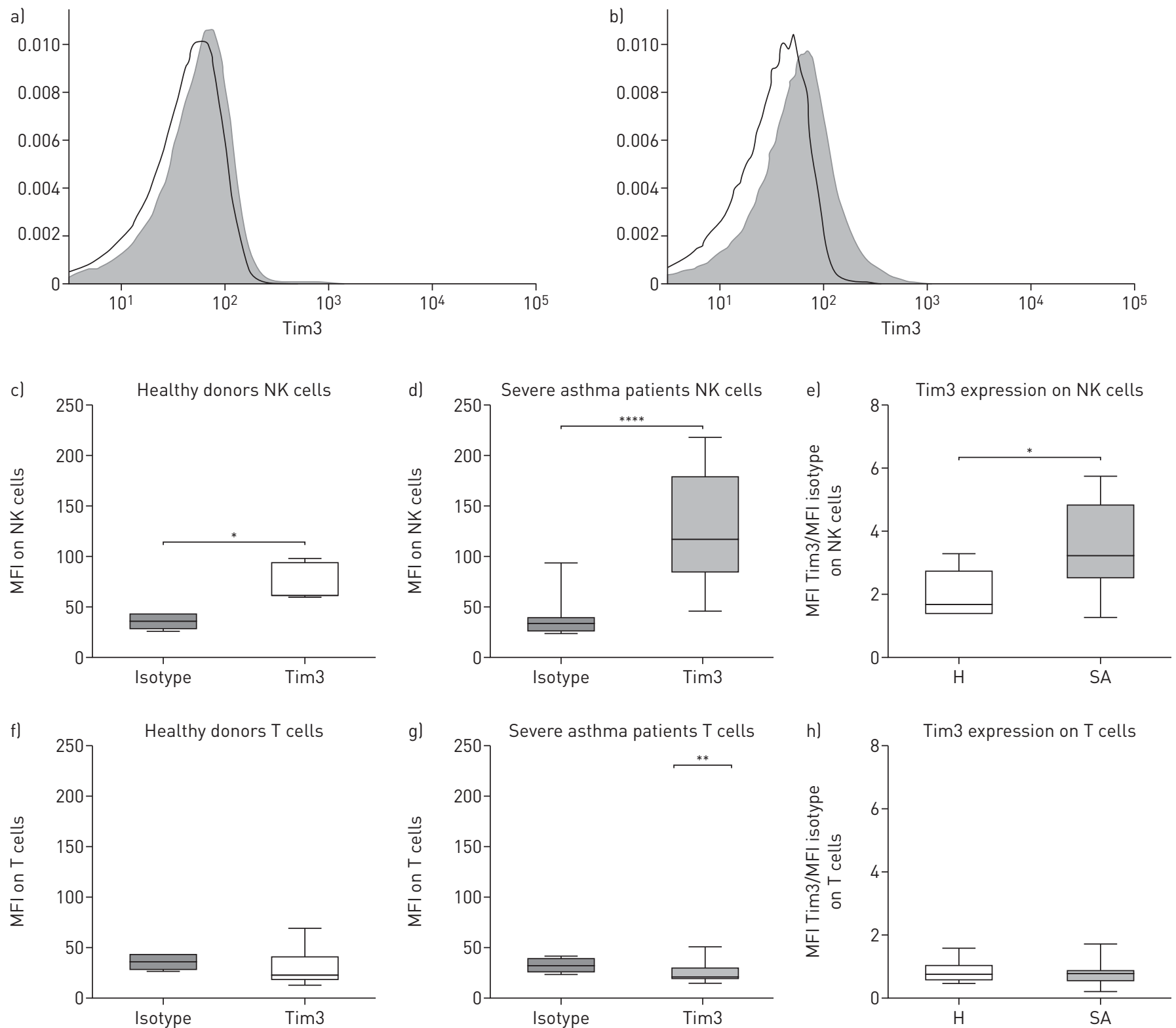

FIGURE 6 Increased Tim3 expression restricted to natural killer (NK) cells from severe asthma patients. Peripheral blood mononuclear cells (PBMC) were obtained from healthy donors $(n=7)$ and severe asthma patients $(n=15)$, and stained with Tim3 specific monoclonal antibody or control isotype antibody. One representative histogram is shown for NK cell staining in (a) for healthy donors and (b) for severe asthma patients, with Tim3 staining in grey and control isotype staining in white. Mean fluorescence intensity (MFI) values are shown for Tim3 and isotype antibodies for NK cells from healthy donors (c) and severe asthma patients (d). Ratio MFI Tim3/MFI Isotype was compared for NK cells between healthy subjects ( $\mathrm{H}$, white) and severe asthma patients (SA, grey) (e). In parallel, MFI values are shown for Tim3 and isotype antibodies for T cells from healthy donors ( $f$ ) and severe asthma patients ( $g$ ). Ratio MFI Tim3/MFI Isotype was compared for $T$ cells between healthy subjects ( $H$, white) and severe asthma patients (SA, grey) (h). Horizontal lines represent the median, boxes represent the interquartile range and whiskers represent the range. ${ }^{*}: p<0.05{ }^{* * * *}: p<0.0001$ (Wilcoxon test for paired isotype versus Tim3, or Mann-Whitney test for difference between healthy and severe asthma subjects).

In order to mimic RV stimulation of human PBMC, we first used synthetic agonists for TLR and cytokines produced during viral infection. Stimulation with IL-12+IL-15 or through TLR involved in nucleic acid recognition led to significant NK cell activation and/or degranulation in both donors. CD69 expression on NK cells was significantly decreased in severe asthma patients only after TLR3 stimulation, whereas CD107 expression was significantly lower after TLR7/8 stimulation, and non-significantly lower after TLR3 and TLR9 stimulation. CD69 was originally described as an early activation marker but may potentially regulate other functions like tissue retention and metabolism of expressing cells [28]. Moreover, non-conditioned tumour-associated NK cells were shown to express a high percentage of the CD69 activation marker but weak cytolytic activities [29], showing that CD69 expression may be dissociated 
from NK cell degranulation. Finally, poly(I:C) administered in mice was found to activate mouse NK cells and to upregulate CD69 expression indirectly through accessory cells. NK cells from mice deficient for MDA5 but not TLR3, two receptors for poly(I:C), exhibited decreased CD69 expression, suggesting that MDA-5 had a predominant role in NK cell CD69 upregulation induced by poly(I:C) [30]. We can therefore hypothesise that MDA-5 signalling might be deficient in accessory cells from severe asthma patients leading to decreased NK cell CD69 expression, in contrast to TLR3, 7/8 and 9 signalling. Impaired NK cell cytotoxic functions after PBMC stimulation with IL-12+IL-15 or TLR involved in RV immune response (TLR3 and TLR7/8) in severe asthma patients, are consistent with studies previously published showing that peripheral blood NK cells from severe asthma patients have impaired killing of K562 myeloid target cells after IL-2 stimulation [18]. We measured granzyme B secretion in PBMC supernatant and found that this molecule, which is released from cytotoxic granules and particularly involved in target cell lysis was less secreted, similarly to decreased CD107a expression (witness of degranulation) in severe asthma patients, but only after TLR3 stimulation. However, discrepancy between CD107a expression and granzyme B secretion was observed for R848 and CpG stimulation, suggesting that other cells among PBMC may release granzyme B as previously published [31], and that this granzyme B secretion is not affected in PBMC from severe asthma patients. Defective cytotoxicity of NK cells from severe asthma patients after stimulation through TLR3 and TLR7/8, both involved in RV immune response, are in agreement with the decreased cytotoxicity (CD107a and granzyme B) observed after activation with RV-A9. The defective cytotoxicity of NK cells is also consistent with the increased viral load observed in asthma patients during infection [32], although the severity of lower respiratory tract symptoms is not related to viral load [33].

Another important function of NK cell consists of cytokine production, and more particularly IFN- $\gamma$ production. Lower IFN- $\gamma$ expression in NK cells and secretion in PBMC were measured in severe asthma patients compared to healthy donors. Similarly, NK cells from patients suffering from atopic dermatitis were shown to express less IFN- $\gamma$ following PBMC stimulation with PMA and ionomycin [34]. Although IFN- $\gamma$ production by NK cells is dependent on accessory cells for TLR or RV stimulation, our results on purified NK cells stimulated with IL12+IL-15 also indicate that the defect in its production is, at least partly, intrinsic i.e. independent of other cells.

As previously shown in vivo [35] or in vitro using another cell type [36], CXCL9 was found increased in the supernatants of PBMC from severe asthma patients after IL-12+IL-15 stimulation. Monocytes may be the source of CXCL9, as previously shown after IL-12+IL-18 stimulation, independently of IFN- $\gamma$ [37]. We could not detect any soluble cytokines known to be involved in NK cell stimulation, like type I IFN, IL-12, IL-15 or IL-18, in PBMC supernatants after TLR or RV-A9 stimulation (data not shown). These cytokines may be already used by cells, not involved, or may stimulate NK cells in their membrane form as shown for IL-15 [38] or for IL-18 which can be released in close vicinity of NK cells [39].

We could not show obvious decreased expression of stimulating receptors on NK cells from severe asthma patients (data not shown). Therefore, we focussed on NK cell exhaustion signs. Immune cell exhaustion describes the status of dysfunction of immune cells, usually under the settings of tumours or chronic infections, characterised by decreased effector functions and associated with increased checkpoint molecules [40]. For example, in HIV-infected patients, polyfunctional CD62L $\mathrm{L}^{+} \mathrm{NK}$ cells express high levels of programmed cell death (PD)-1, NKG2A, and T-cell immunoglobulin and mucin-domain containing (Tim)-3. The expression of these markers on NK cell surface is associated with a decreased cytotoxicity and IFN- $\gamma$ expression in response to TLR3 stimulation [40]. Here we showed that the expression of Tim-3 by NK cells was higher in severe asthma patients compared to healthy donors. The expression levels of PD-1 and NKG2A on NK cells were similar between severe asthma patients and healthy donors. Overexpression of these three checkpoint molecules has been associated with NK cell exhaustion and dysfunction in chronic hepatitis B virus (HBV) and HIV infection. However, although impaired TLR3 response of NK cells was shown to be associated with increased expression of NKG2A, PD-1 and Tim-3 in chronic exposure to HIV [40], in HBV chronic infection only Tim-3 over-expression was associated with reduced function of NK cells [41]. Together with our results, these data suggest that NK cell dysfunction may be associated with differential sustained over-expression of checkpoint molecules depending on the pathology. In severe asthma, we can hypothesise that chronic inflammation may induce NK cell dysfunction in particular through Tim-3 signalling, leading to inefficient activation of NK cells in response to RV-A9. The effect of Tim-3 blockade on restoring NK cell activity needs to be further explored as previously shown in chronic virus infection [42] or cancer [43] setting.

The involvement of corticosteroids in NK cell defect in severe asthma needs to be questioned. Indeed, treatments for severe asthma are mainly constituted with high doses of inhaled and/or systemic corticosteroids as anti-inflammatory agents. It was shown that intramuscular injection of triamcinolone modifies BAL NK cell phenotype, but not BAL IFN- $\gamma$ level. Furthermore, in vitro treatment of PBMC with 
dexamethasone significantly inhibits the capacity of NK cells to lyse K562 target cells, both in healthy donors and severe asthma patients [18]. In our study, all severe asthma patients were treated with inhaled corticosteroids, and $45.2 \%$ of them received systemic corticosteroids. The cytotoxicity and IFN- $\gamma$ expression were not different between patients with and without systemic corticosteroids. However, statistical power was insufficient to conclude to a true absence of difference. We analysed Tim-3 expression on NK cells after in vitro treatment of PBMC isolated from three different healthy donors with increasing doses of dexamethasone. Dexamethasone did not increase Tim-3 expression for the 10 and $100 \mathrm{ng} \cdot \mathrm{mL}^{-1}$ doses. Moreover, PBMC in vitro stimulation with a different RV, this one belonging to the minor-group (i.e. RV-2), induced NK cell activation levels (increased CD69, CD107 and IFN- $\gamma$ expression) similar between severe asthma patients and healthy subjects. This result may seem puzzling. However, exposure of primary monocytic cells to two genetically similar serotypes of RV, one major and one minor group, was shown to elicit differential activation of signalling molecules and transcription factors leading to different responses [44]. Our observation suggests that RV serotypes may lead to differential NK cell activation depending on the patient status, but further studies comparing several strains of each group are needed to conclude. Moreover, our results suggest that there is not a general viral deficient response to rhinoviruses but rather that NK cells from severe asthma patients responds with a virus-specific signalling response. Moreover, this result further emphasises that a defect in NK cells may be related to the severe asthmatic status rather than to the treatment, although corticosteroids may certainly modify NK cell-induced immune response in severe asthma patients, and therefore have adverse effects in RV-induced exacerbations.

In conclusion, we found that NK cells from severe asthma patients have impaired in vitro functions in response to one serotype of rhinovirus, and some virus-mimicking molecules (TLR3, TLR7/8 agonists). This defect may be linked to NK cell exhaustion and may contribute to the severity of virus-induced asthma exacerbations in severe asthma patients. NK cell exhaustion may also be responsible for decreased cytotoxicity of NK cells from severe asthma patients towards eosinophils [18]. Therefore, our study emphasises that aberrant NK cell functions may participate to the severity and virus-induced exacerbation of asthma.

Acknowledgements: We thank BioImaging Center Lille Nord-de-France for access to a flow cytometer, H. Bauderlique and C. Faveeuw for technical advice in flow cytometry, P. Gosset for critically reviewing the manuscript, C. Lecoeur for statistical advice (all from Center for Infection and Immunity of Lille, France), and G. Duverlie for providing RV-2 (Laboratoire de virologie, CHU Amiens Picardie, France).

Conflict of interest: J. Devulder has a patent EP18306287.6 issued to INSERM. C. Chenivesse has a patent EP18306287.6 issued to INSERM. V. Ledroit has nothing to disclose. S. Fry has nothing to disclose. P-E. Lobert has nothing to disclose. D. Hober has nothing to disclose. A. Tsicopoulos has nothing to disclose. C. Duez has a patent EP18306287.6 issued to INSERM.

Support statement: This work was supported by INSERM. J. Devulder is supported by a grant from Ministère de l'Enseignement supérieur, de la Recherche et de l'Innovation, and by a grant from Fonds de Recherche en Santé Respiratoire (FRSR). Funding information for this article has been deposited with the Crossref Funder Registry.

\section{References}

1 Lambrecht BN, Hammad H. The immunology of asthma. Nat Immunol 2014; 16: 45-56.

2 Chung KF, Wenzel SE, Brozek JL, et al. International ERS/ATS guidelines on definition, evaluation and treatment of severe asthma. Eur Respir J 2014; 43: 343-373.

3 Stone B, Davis JR, Trudo F, et al. Characterizing patients with asthma who received Global Initiative for Asthma steps 4-5 therapy and managed in a specialty care setting. Allergy Asthma Proc 2018; 39: 27-35.

4 Chastek B, Korrer S, Nagar SP, et al. Economic burden of illness among patients with severe asthma in a managed care setting. J Manag Care Spec Pharm 2016; 22: 848-861.

5 Dalal AA, Duh MS, Gozalo L, et al. Dose-response relationship between long-term systemic corticosteroid use and related complications in patients with severe asthma. J Manag Care Spec Pharm 2016; 22: 833-847. McDonald VM, Gibson PG. Exacerbations of severe asthma. Clin Exp Allergy 2012; 42: 670-677.

Sykes A, Johnston SL. Etiology of asthma exacerbations. J Allergy Clin Immunol 2008; 122: 685-688. Edwards MR, Bartlett NW, Hussell T, et al. The microbiology of asthma. Nat Rev Microbiol 2012; 10: $459-471$. Edwards MR, Strong K, Cameron A, et al. Viral infections in allergy and immunology: How allergic inflammation influences viral infections and illness. J Allergy Clin Immunol 2017; 140: 909-920.

10 Laza-Stanca V, Message SD, Edwards MR, et al. The role of IL-15 deficiency in the pathogenesis of virus-induced asthma exacerbations. PLoS Pathog 2011; 7: e1002114.

11 Jacobs SE, Lamson DM, St George K, et al. Human rhinoviruses. Clin Microbiol Rev 2013; 26: $135-162$.

12 Vivier E, Tomasello E, Baratin M, et al. Functions of natural killer cells. Nat Immunol 2008; 9: 503-510.

13 Long EO, Sik Kim H, Liu D, et al. Controlling natural killer cell responses: integration of signals for activation and inhibition. Annu Rev Immunol 2013; 31: 227-258.

14 Mandelboim O, Lieberman N, Lev M, et al. Recognition of haemagglutinins on virus-infected cells by NKp46 activates lysis by human NK cells. Nature 2001; 409: 1055-1060.

15 Adib-Conquy M, Scott-Algara D, Cavaillon J-M, et al. TLR-mediated activation of NK cells and their role in bacterial/viral immune responses in mammals. Immunol Cell Biol 2014; 92: 256-262. 
16 Caligiuri MA. Human natural killer cells. Blood 2008; 112: 461-469.

17 Barnig C, Cernadas M, Dutile S, et al. Lipoxin A4 regulates natural killer cell and type 2 innate lymphoid cell activation in asthma. Sci Transl Med 2013; 5: 174ra26.

18 Duvall MG, Barnig C, Cernadas M, et al. Natural killer cell-mediated inflammation resolution is disabled in severe asthma. Sci Immunol 2017; 2: eaam5446.

19 Lin S-J, Chang L-Y, Yan D-C, et al. Decreased intercellular adhesion molecule-1 (CD54) and L-selectin (CD62L) expression on peripheral blood natural killer cells in asthmatic children with acute exacerbation. Allergy 2003; 58: $67-71$.

20 Awad A, Yassine H, Barrier M, et al. Natural killer cells induce eosinophil activation and apoptosis. PLoS ONE 2014; 9: e94492.

21 Ghadially H, Horani A, Glasner A, et al. NKp46 regulates allergic responses. Eur J Immunol 2013; 43: 3006-3016.

22 Korsgren M, Persson CGA, Sundler F, et al. Natural killer cells determine development of allergen-induced eosinophilic airway inflammation in mice. J Exp Med 1999; 189: 553-562.

23 Haspeslagh E, van Helden MJ, Deswarte K, et al. Role of $\mathrm{NKp}^{+} 6^{+}$natural killer cells in house dust mite-driven asthma. EMBO Mol Med 2018; 10: e8657.

24 Haworth O, Cernadas M, Levy BD. NK cells are effectors for resolvin E1 in the timely resolution of allergic airway inflammation. J Immunol 2011; 186: 6129-6135.

25 Simons B, Ferrini ME, Carvalho S, et al. $\mathrm{PGI}_{2}$ controls pulmonary NK cells that prevent airway sensitization to house dust mite allergen. J Immunol 2017; 198: 461-471.

26 Ferrini ME, Hong S, Stierle A, et al. CB2 receptors regulate natural killer cells that limit allergic airway inflammation in a murine model of asthma. Allergy 2017; 72: 937-947.

27 Lunding LP, Webering S, Vock C, et al. Poly(inosinic-cytidylic) acid-triggered exacerbation of experimental asthma depends on IL-17A produced by NK cells. J Immunol 2015; 194: 5615-5625.

28 Cibrián D, Sánchez-Madrid F. CD69: from activation marker to metabolic gatekeeper. Eur J Immunol 2017; 47: 946-953.

29 Bellora F, Castriconi R, Dondero A, et al. TLR activation of tumor-associated macrophages from ovarian cancer patients triggers cytolytic activity of NK cells. Eur J Immunol 2014; 44: 1814-1822.

30 McCartney S, Vermi W, Gilfillan S, et al. Distinct and complementary functions of MDA5 and TLR3 in poly(I: C)-mediated activation of mouse NK cells. J Exp Med 2009; 206: 2967-2976.

31 Boivin WA, Cooper DM, Hiebert PR, et al. Intracellular versus extracellular granzyme B in immunity and disease: challenging the dogma. Lab Investig J Tech Methods Pathol 2009; 89: 1195-1220.

32 Kennedy JL, Shaker M, McMeen V, et al. Comparison of viral load in individuals with and without asthma during infections with rhinovirus. Am I Respir Crit Care Med 2014; 189: 532-539.

33 van Elden LJR, Sachs APE, van Loon AM, et al. Enhanced severity of virus associated lower respiratory tract disease in asthma patients may not be associated with delayed viral clearance and increased viral load in the upper respiratory tract. J Clin Virol Off Publ Pan Am Soc Clin Virol 2008; 41: 116-121.

34 Luci C, Gaudy-Marqueste C, Rouzaire P, et al. Peripheral natural killer cells exhibit qualitative and quantitative changes in patients with psoriasis and atopic dermatitis. Br J Dermatol 2012; 166: 789-796.

35 Hosoki K, Ying S, Corrigan C, et al. Analysis of a panel of 48 cytokines in BAL fluids specifically identifies IL-8 levels as the only cytokine that distinguishes controlled asthma from uncontrolled asthma, and correlates inversely with FEV1. PLoS One 2015; 10: e0126035.

36 Tan KS, Ong HH, Yan Y, et al. In vitro model of fully differentiated human nasal epithelial cells infected with rhinovirus reveals epithelium-initiated immune responses. J Infect Dis 2018; 217: 906-915.

37 Coma G, Peña R, Blanco J, et al. Treatment of monocytes with interleukin (IL)-12 plus IL-18 stimulates survival, differentiation and the production of CXC chemokine ligands (CXCL)8, CXCL9 and CXCL10. Clin Exp Immunol 2006; 145: 535-544.

38 Ferlazzo G, Pack M, Thomas D, et al. Distinct roles of IL-12 and IL-15 in human natural killer cell activation by dendritic cells from secondary lymphoid organs. Proc Natl Acad Sci USA 2004; 101: 16606-16611.

39 Bellora F, Castriconi R, Doni A, et al. M-CSF induces the expression of a membrane-bound form of IL-18 in a subset of human monocytes differentiating in vitro toward macrophages. Eur J Immunol 2012; 42: 1618-1626.

40 Lima JF, Oliveira LMS, Pereira NZ, et al. Polyfunctional natural killer cells with a low activation profile in response to Toll-like receptor 3 activation in HIV-1-exposed seronegative subjects. Sci Rep 2017; 7: 524

41 Li X, Zhou L, Gu L, et al. Veritable antiviral capacity of natural killer cells in chronic HBV infection: an argument for an earlier anti-virus treatment. J Transl Med 2017; 15: 220.

42 Ju Y, Hou N, Meng J, et al. T cell immunoglobulin- and mucin-domain-containing molecule-3 (Tim-3) mediates natural killer cell suppression in chronic hepatitis B. J Hepatol 2010; 52: 322-329.

43 Gallois A, Silva I, Osman I, et al. Reversal of natural killer cell exhaustion by TIM-3 blockade. OncoImmunology 2014; 3: e946365.

44 Schuler BA, Schreiber MT, Li L, et al. Major and minor group rhinoviruses elicit differential signaling and cytokine responses as a function of receptor-mediated signal transduction. PLoS One 2014; 9: e93897. 